\title{
ON THE SOLUTION OF REACTION-DIFFUSION EQUATIONS WITH DOUBLE DIFFUSIVITY
}

\author{
B.D. AGGARWALA and C. NASIM \\ Department of Mathematics and Statistics \\ The University of Calgary \\ Calgary, Alberta TIN IN4 Canada
}

(Received September 9, 1985 and in revised form March 28, 1986)

ABSTRACT. In this paper, solution of a pair of Coupled Partial Differential equations is derived. These equations arise in the solution of problems of flow of homogeneous liquids in fissured rocks and heat conduction involving two temperatures. These equations have been considered by $\mathrm{Hill}$ and $\mathrm{Aifantis,} \mathrm{but} \mathrm{the} \mathrm{technique} \mathrm{we} \mathrm{use} \mathrm{appears} \mathrm{to}$ be simpler and more direct, and some new results are derived. Also, discussion about the propagation of initial discontinuities is given and illustrated with graphs of some special cases.

KEY WORDS AND PHRASES. Reaction Diffusion equations, Fluid Flow in Fissured rocks, Laplace and Fourier Transforms, Propagation of Initial Discontinuities.

Subject Classification: $35 \mathrm{~K} 57$

1. INTRODUCTION.

In this paper we solve a pair of coupled Partial Differential Equations, which pair may arise in a number of physical situations, including, e.g., flow of homogeneous liquids in fissured rocks [1] and heat conduction involving two temperatures [2]. Such equations have been considered by Hill [3], by Hill and Aifantis [4,5] and by Lee and Hill [6], wherein they have arrived at the same solutions as those in this paper, albeit by a different technique. Our method, in which inversion depends upon the idea behind equation (3.5), appears to be simpler and more direct than that of these investigators. Also, we have deduced some results from these solutions which are not derived in literature. See also Gopalsamy and Aggarwala [7] where a slightly different pair of coupled partial differential equations is considered in a different setting.

2. THE PROBLEM

We wish to find solutions $u(x, t)$ and $v(x, t)$ of the equations

$$
\begin{aligned}
& \frac{\partial u}{\partial t}=D_{1} \nabla^{2} u-a_{1} u+b_{1} v \\
& \frac{\partial v}{\partial t}=D_{2} \nabla^{2} v+a_{2} u-b_{2} v
\end{aligned}
$$

(where $a_{1}, b_{1}, a_{2}$ and $b_{2}$ are constants), with the initial conditions

$$
u=f(x) \text { and } v=0 \text { at } t=0 \text {, and with (identical) }
$$

homogeneous boundary conditions on $u$ and $v$. 
3. SOLUTION

Taking the Laplace Transform $(u(x, t) \rightarrow \bar{u}(x, s))$ of $(2.1)$, we get

$$
\begin{aligned}
& s \bar{u}=D_{1} \nabla^{2} \bar{u}-a_{1} \bar{u}+b_{1} \bar{v}+f(x) \\
& s \bar{v}=D_{2} \nabla^{2} \bar{v}+a_{2} \bar{u}-b_{2} \bar{v} .
\end{aligned}
$$

We now assume that there is a Fourier Transform $(\bar{u}(x, s) \rightarrow \overline{\bar{u}}(\xi, s))$ such that $\bar{\nabla} \overline{\mathrm{u}}=-\xi \overline{\mathrm{u}}$. Taking this Fourier Transform, we get

$$
\begin{aligned}
& s \overline{\bar{u}}=-D_{1} \xi^{2 \overline{\bar{u}}}-a_{1} \overline{\bar{u}}+b_{1} \overline{\bar{v}}+\vec{f}(\xi) \\
& s \overline{\bar{v}}=-D_{2} \bar{\xi}^{2 \bar{v}}+a_{2} \overline{\bar{u}}-b_{2} \overline{\bar{v}}
\end{aligned}
$$

where $\bar{f}(\xi)$ is the Fourier Transform of $f(x)$.

These equations give

$$
\overline{\bar{v}}=\frac{a_{2} \bar{f}(\xi)}{D_{1} D_{2}\left(\xi^{2}+\xi_{1}^{2}\right)\left(\xi^{2}+\xi_{2}^{2}\right)}
$$

where

$$
\begin{aligned}
& \xi_{1,2}^{2}=\frac{\left[\left(s+a_{1}\right) D_{2}+\left(s+b_{2}\right) D_{1}\right]}{-\sqrt{\left[\left(s+a_{1}\right) D_{2}-\left(s+b_{2}\right) D_{1}\right]^{2}+4 D_{1} D_{2} b_{1} a_{2}}} \\
& =A \mp \sqrt{C^{2}+B^{2}} \text {, say. }
\end{aligned}
$$

To invert $\overline{\bar{v}}$, consider the problem $\frac{\partial h}{\partial t}=\nabla^{2} h$ with $h=f(x)$ at $t=0$ and the same boundary conditions as on $u$ and $v$.

Taking the Laplace and Fourier Transforms, we get

$$
\overline{\bar{h}}=\frac{\overline{\mathbf{f}}(\boldsymbol{\xi})}{\mathbf{s}+\xi^{2}} .
$$

If we now consider the fact that the inverse Fourier Transform of $\bar{h}$ is given by $\int_{0}^{\infty} e^{-s t} h(x, t) d t$, then since

$$
\overline{\bar{v}}=\frac{\mathrm{a}_{2}}{\mathrm{D}_{1} \mathrm{D}_{2}\left(\xi_{2}^{2}-\xi_{1}^{2}\right)}\left[\frac{\overline{\mathrm{f}}(\xi)}{\xi^{2}+\xi_{1}^{2}}-\frac{\overline{\mathrm{f}}(\xi)}{\xi^{2}+\xi_{2}^{2}}\right]
$$

it follows that the inverse Fourier Transform of $\overline{\bar{v}}$ is given by

$$
\begin{aligned}
& \bar{v}=\frac{a_{2}}{D_{1} D_{2}\left(\xi_{2}^{2}-\xi_{1}^{2}\right)} \int_{0}^{\infty}\left[e^{-\xi_{1}^{2} t}-e^{-\xi_{2}^{2} t}\right] h(x, t) d t \\
&=\frac{a_{2}}{D_{1} D_{2}} \int_{0}^{\infty} e^{-A t} \frac{\sinh \left(t \sqrt{B^{2}+C^{2}}\right.}{\sqrt{B^{2}+C^{2}}} h(x, t) d t \\
&=\frac{a_{2}}{D_{1} D_{2}} \int_{0}^{\infty} \int_{0}^{\pi / 2} e^{-A t} h(x, t) I_{0}(B t \sin \theta) \cosh (C t \cos \theta) t \sin \theta d \theta d t
\end{aligned}
$$

(see [8], p.743(2))

$=\frac{a_{2}}{2 D_{1} D_{2}} \int_{0}^{\infty} \int_{0}^{\pi / 2}\left[e^{-s k} 1^{u} \cdot e^{-k_{3} u}+e^{-s k} 2^{u} \cdot e^{-k} 4^{u}\right] h(x, u) I_{0}(B u \sin \theta) u \sin \theta d \theta d u$

where

$$
\begin{gathered}
k_{1,2}=\frac{D_{1}+D_{2}}{2 D_{1} D_{2}} \mp \frac{D_{1}-D_{2}}{2 D_{1} D_{2}} \cos \theta \\
k_{3,4}=\frac{D_{1} b_{2}+D_{2} a_{1}}{2 D_{1} D_{2}} \mp \frac{D_{1} b_{2}-D_{2} a_{1}}{2 D_{1} D_{2}} \cos \theta .
\end{gathered}
$$


The inverse Laplace Transform must now be performed w.r.t. $s(s \rightarrow t)$ and noting that, with the usual notation,

$$
\int_{0}^{\infty} e^{-s t} \delta(t-\xi) d t=e^{-s \xi}
$$

(where $\delta$ is the Dirac Delta function), it is given by

$v=\frac{a_{2}}{2 D_{1} D_{2}} \int_{0}^{\infty} \int_{0}^{\pi / 2}\left[e^{-k_{3} u} \delta\left(t-u k_{1}\right)+e^{-k_{4} u} \delta\left(t-u k_{2}\right)\right] I_{0}(B u \sin \theta) h(x, u) u \sin \theta d \theta d u .(3.10)$ Using $\int_{0}^{\infty} f(u) \delta\left(t-k_{1} u\right) d u=\frac{1}{k_{1}} f\left[\frac{t}{k_{1}}\right]$, we get

$$
\begin{aligned}
& v=\frac{a_{2}}{2 D_{1} D_{2}} \int_{0}^{\pi / 2} \frac{t}{k_{1}^{2}} e^{-k_{3} t / k_{1}} I_{0}\left(t B \sin \theta / k_{1}\right) h\left(x, t / k_{1}\right) \sin \theta d \theta \\
& +\frac{a_{2}}{2 D_{1} D_{2}} \int_{0}^{\pi / 2} \frac{t}{k_{2}^{2}} e^{-k_{4} t / k_{2}} I_{0}\left(t B \sin \theta / k_{2}\right) h\left(x, t / k_{2}\right) \sin \theta d \theta \\
& =I_{1}+I_{2} .
\end{aligned}
$$

Putting $u=\frac{1}{k_{1}}$ in $I_{1}, u=\frac{1}{k_{2}}$ in $I_{2}$, and combining the two integrals, we get

$$
v=\frac{a_{2} t e^{-\delta t}}{D_{1}-D_{2}} \int_{D_{2}}^{D_{1}} e^{-r u t} I_{0}(n t) h(x, u t) d u
$$

where

$$
\begin{gathered}
r=\frac{a_{1}-b_{2}}{D_{1}-D_{2}}, \quad \delta=\frac{D_{1} b_{2}-a_{1} D_{2}}{D_{1}-D_{2}} \text { and } \\
n=\frac{2 \sqrt{a_{2} b_{1}}}{\mid D_{1}-D_{2}} \sqrt{T\left(D_{1}-u\right)\left(u-D_{2}\right) T .}
\end{gathered}
$$

Now, using (2.1b) and the fact that $\nabla^{2} h=o h / o t$, we get, after some simplification

$$
u=e^{-a_{1} t} h\left(x, D_{1} t\right)+\frac{\sqrt{b_{1} a_{2}} e^{-\delta t}}{D_{1}-D_{2}} \int_{D_{2}}^{D_{1}} t e^{-r u t} I_{1}(n t) h(x, u t) \sqrt{\frac{u-D_{2}}{D_{1}-u} d u . ~}
$$

Equations (3.12), (3.13) and (3.14) give the solution to our problem.

It may be noted that equations (3.5) and (3.1b) may be used to invert these relations [3].

4. SPECIAL CASE

The most important case for applications is when $a_{1}=a_{2}=b_{1}=b_{2}=a$. In this case the solution becomes

$$
v=\frac{\alpha e^{-\alpha t}}{D_{1}-D_{2}} \int_{D_{2}}^{D_{1}} t h(x, u t) I_{0}(n t) d u
$$

and

$$
u=e^{-\alpha t} h\left(x, D_{1} t\right)+\frac{\alpha e^{-\alpha t}}{D_{1}-D_{2}} \int_{D_{2}}^{D_{1}} t h(x, u t) I_{1}(n t) \sqrt{\frac{u-D_{2}}{D_{1}-u}} d u
$$

where

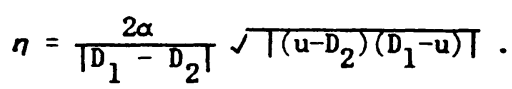


If, in this case, we assume that $\int h(x, t) d x=1$ for all $t$, we readily obtain

$$
\begin{aligned}
& \int v d x=e^{-\alpha t} \sinh \alpha t \\
& \int u d x=e^{-\alpha t} \cosh \alpha t
\end{aligned}
$$

which shows that if initially the "u-system" is supplied with a "unit" of heat and no heat is escaping from the boundaries (the boundary conditions on $h$ are the same as those on $u$ or $v$ ) then half of the heat goes into the "u-system" and the other half goes into the "v-system" at the rate implied by equations (4.2). It is interesting to note that this result is independent of the relative magnitudes of $D_{1}$ and $D_{2}$.

In many cases $h(x, t)$ is given as a sum (or integral) of a function of $x$ multiplied by a function of $t$. If we assume

$$
h(x, t)=\sum_{\beta} F_{\beta}(x) e^{-\beta t}
$$

integration gives

and

$$
\begin{gathered}
u=e^{-\alpha t} h\left(x, D_{1} t\right)+e^{-\alpha t} \sum_{\beta} F_{\beta}(x) G_{\beta}(t) \\
v=e^{-\alpha t} \sum_{\beta} F_{\beta}(x) H_{\beta}(t)
\end{gathered}
$$

where

$$
\begin{gathered}
G_{\beta}(t)=e^{-\beta t\left(D_{1}+D_{2}\right) / 2}\left[\cosh \left(t \sqrt{A_{1}^{2}+A_{2}^{2}}\right)-\frac{A_{1}}{\sqrt{A_{1}^{2}+A_{2}^{2}}} \sinh \left(t \sqrt{A_{1}^{2}+A_{2}^{2}}\right)-e^{-A_{1} t}\right] \\
H_{\beta}(t)=\alpha e^{-\beta t\left(D_{1}+D_{2}\right) / 2 \sinh \left(t \sqrt{A_{1}^{2}+A_{2}^{2}}\right)}
\end{gathered}
$$

where

$$
A_{1}=\frac{\beta}{2}\left(D_{1}-D_{2}\right), A_{2}=\alpha .
$$

To obtain equations (4.3), we need

$$
I_{1}=\int_{D_{2}}^{D_{1}} e^{-\beta u t} I_{1}(n t) \sqrt{\frac{u-D_{2}}{D_{1}-u}} d u
$$

and

$$
I_{2}=\int_{D_{2}}^{D_{1}} e^{-\beta u t} I_{0}(n t) d u
$$

where $n$ is given by (4.1c).

If we put $u=D_{1} \sin ^{2} \theta+D_{2} \cos ^{2} \theta$

we get

and

$$
I_{1}=\frac{D_{1}-D_{2}}{2} e^{-\beta t\left(D_{1}+D_{2}\right) / 2}\left(I_{3}-I_{4}\right)
$$

$$
I_{2}=2 \int_{0}^{\pi / 2} \cosh \left(\Lambda_{1} t \cos x\right) I_{0}\left(A_{2} t \sin x\right) \sin x d x
$$

where

$$
I_{3}=2 \int_{0}^{\pi / 2} \cosh \left(A_{1} t \cos x\right) I_{0}\left(A_{2} t \sin x\right) d x
$$

and 


$$
I_{4}=2 \int_{0}^{\pi / 2} \sinh \left(A_{1} t \cos x\right) I_{1}\left(A_{2} t \sin x\right) \cos x d x .
$$

These integrals may be evaluated with the help of known results (see, e.g. [8] pages $742(1)$ and $743(2))$.

\section{SEPARATION OF VARIABLES}

It is to be noted that equations (4.3) may also be derived by assuming the solution to be of the type $u=F_{\beta}(x) G_{\beta}(t), v=F_{\beta}(x) H_{\beta}(t)$ where $F_{\beta}(x)$ are solutions of $\nabla^{2} F_{\beta}+\beta F_{\beta}=0$, solving the resultant pair of ordinary differential equations in $G_{\beta}$ and $H_{\beta}$ with $G_{\beta}=1, H_{\beta}=0$ at $t=0$ and superposing. The same process may also be used to solve equations (2.1), in which case the results are

$$
\begin{gathered}
u=e^{-a_{1} t} h\left(x, D_{1} t\right)+\sum_{\beta} F_{\beta}(x) G_{\beta}(t) \\
v=\sum_{\beta} F_{\beta}(x) H_{\beta}(t)
\end{gathered}
$$

where

$$
\begin{gathered}
\sum F_{\beta}(x)=f(x), \quad h(x, t)=\Sigma F_{\beta}(x) e^{-\beta t} \\
H_{\beta}(t)=\frac{a_{2}}{\sqrt{A_{1}^{2}+A_{2}^{2}}} e^{-k t} \sinh \left(t \sqrt{A_{1}^{2}+A_{2}^{2}}\right) \\
G_{\beta}(t)=e^{-k t} \cosh \left(t \sqrt{A_{1}^{2}+A_{2}^{2}}\right)-\frac{A_{1}}{\sqrt{A_{1}^{2}+A_{2}^{2}}} e^{-k t} \sinh \left(t \sqrt{A_{1}^{2}+A_{2}^{2}}\right)-e^{-\left(a_{1}+\beta D_{1}\right) t}
\end{gathered}
$$

where now

$$
\begin{gathered}
k=\left(D_{1} \beta+D_{2} \beta+a_{1}+b_{2}\right) / 2 \\
A_{1}=\left(D_{1} \beta+a_{1}-D_{2} \beta-b_{2}\right) / 2 \\
A_{2}=\sqrt{a_{2} b_{1}} .
\end{gathered}
$$

6. PROPAGATION OF INITIAL DISCONTINUITIES

It is to be noticed that the last term in $G_{\beta}(t)$ simply cancels out the first term in $u$. We shall disregard this term in $G_{\beta}(t)$ in this discussion. If now $D_{1} D_{2} \neq 0$, then the initial discontinuities of $u$ and $v$ are immediately smoothed out. If $D_{1} \neq 0$ and $D_{2}=0$, then for large values of $\beta$ in $(5.1), H_{\beta}(t) \approx e^{-b_{2} t} / \beta$ and $G_{\beta}(t) \simeq e^{-b_{2} t} / \beta^{2}$, so that if $\sum F_{\beta}(x)(=f(x))$ is discontinuous at some point, then this initial discontinuity of $u$ is smoothed out for $t>0$. If, however, $D_{1}=0$ and $D_{2} \neq 0$, then for large values of $\beta, H_{\beta}(t) \simeq e^{-a_{1} t} / \beta$ and $G_{\beta}(t)=e^{-a_{1} t}+0\left(\frac{1}{\beta}\right)$, so that, while the initial discontinuities of $u$ do not get propagated into $v$, they stay as discontinuities of $u$ and decay exponentially as $e^{-a_{1} t}$. The same statement is also true for discontinuities in normal derivatives across some surface.

If $D_{1}=0$, and we eliminate $v$ from equations (2.1), we get

$$
\frac{\partial^{2} \mathrm{u}}{\partial \mathrm{t}^{2}}-\mathrm{D}_{2} \frac{\partial}{\partial \mathrm{t}} \nabla^{2} \mathrm{u}-\mathrm{a}_{1} \mathrm{D}_{2} \nabla^{2} \mathrm{u}+\left(\mathrm{a}_{1}+\mathrm{b}_{2}\right) \frac{\partial \mathrm{u}}{\partial \mathrm{t}}+\left(\mathrm{a}_{1} \mathrm{~b}_{2}-\mathrm{a}_{2} \mathrm{~b}_{1}\right) \mathrm{u}=0
$$

If now we prescribe the initial values of $u$ and $\frac{\partial u}{\partial t}$ and the boundary values of $u$ for 
(6.1) and if the initial values of $u$ as we approach the boundary do not coincide with the boundary values of $u$ as we approach $t=0$, then there is an initial discontinuity in $u$ near the boundary which does not die out immediately. The boundary condition on $u$ in this case should be modified from the consideration that for $D_{2} \neq 0$, there are no discontinuities in $v$ and then, integration of (2.la) gives

$$
u(s, t)=u(s, 0) e^{-a_{1} t}+e^{-a_{1} t} \int_{0}^{t} e^{a_{1}{ }^{\theta}} b_{1} v(s, \theta) d \theta
$$

where $s$ is a point on the boundary. If, e.g., $u(x, 0)=P_{0}, u(s, t)=P_{1}$ for $t>0$ (which, using (2.la), gives $b_{1} v(s, t)=a_{1} P_{1}$ for $\left.t>0\right),(6.2)$ gives

$$
u(s, t)=P_{1}+\left(P_{0}-P_{1}\right) e^{-a_{1} t} \text {. }
$$

This equation once again indicates that the initial discontinuities of $u\left(P_{0}-P_{1}\right.$ in this case) die out exponentially as $e^{-a_{1} t}$. The result in equation (6.3) coincides with the one obtained in [1] for the case when $\frac{\partial v}{\partial t}$ term is absent in $(2.1 b)$ and $D_{1}=0$. In equation (6.2), $u(s, 0)=\operatorname{Lim}_{x \rightarrow s} u(x, 0)$. In (6.3), $P_{0}$ and $P_{1}$ are constants. Also $f(x)$ (or $\partial \mathrm{f} / \partial \mathrm{n}$ in the case of possible discontinuities in the normal derivatives) in the above discussion is assumed to be such that $\Sigma F_{\beta}(x) / \beta$ (or $\Sigma\left(\partial F_{\beta}(x) / \partial n\right) / \beta$ respectively) is uniformly convergent. The symbol $x$ denotes a vector in $n$-dimensions throughout, however the following examples and graphs are given for $n=1$.

Examples: We give some examples to illustrate the above results. Numerical values are indicated in Figs. 1-6. $a_{1}=a_{2}=b_{1}=b_{2}=1$.

1. In this case $f(x)=x / 2$ for $0 \leq x<.5$ and $f(x)=\frac{1-x}{2}$ for $.5<x \leq 1$. $D_{1}=0$ and $D_{2}=1$. Fig. 1 illustrates the decay of discontinuity in $\partial u / \partial x$ at $x=.5$.

2. $f(x)$ is the same as in example $1 . D_{1}=1, D_{2}=0$. Fig. 2 illustrates the smoothing of discontinuity for $t>0$.

3. $f(x)=0$ for $0 \leq x<.5$ and $f(x)=1$ for $.5<x \leq 1 . u=v=0$ at $x=0$ and $x=1$. Fig. 3 illustrates the decay of discontinuity in $u$ at $x=.5$ and the modification of boundary value at $x=1 . D_{1}=0, D_{2}=1$.

4. Same as example 3 but with $D_{1}=1, D_{2}=0$. Discontinuities are smoothed out in Fig. 4.

5. In this case $f(x)=1-\cos \pi x, \partial u / \theta n=0$ at $x=0$ and $x=1$. Fig. 5 illustrates $u \rightarrow 1 / 2, v \rightarrow 1 / 2$ as $t \rightarrow \infty . D_{1}=0, D_{2}=1$.

6. Same as example 5 with $D_{1}=1, D_{2}=0$. 


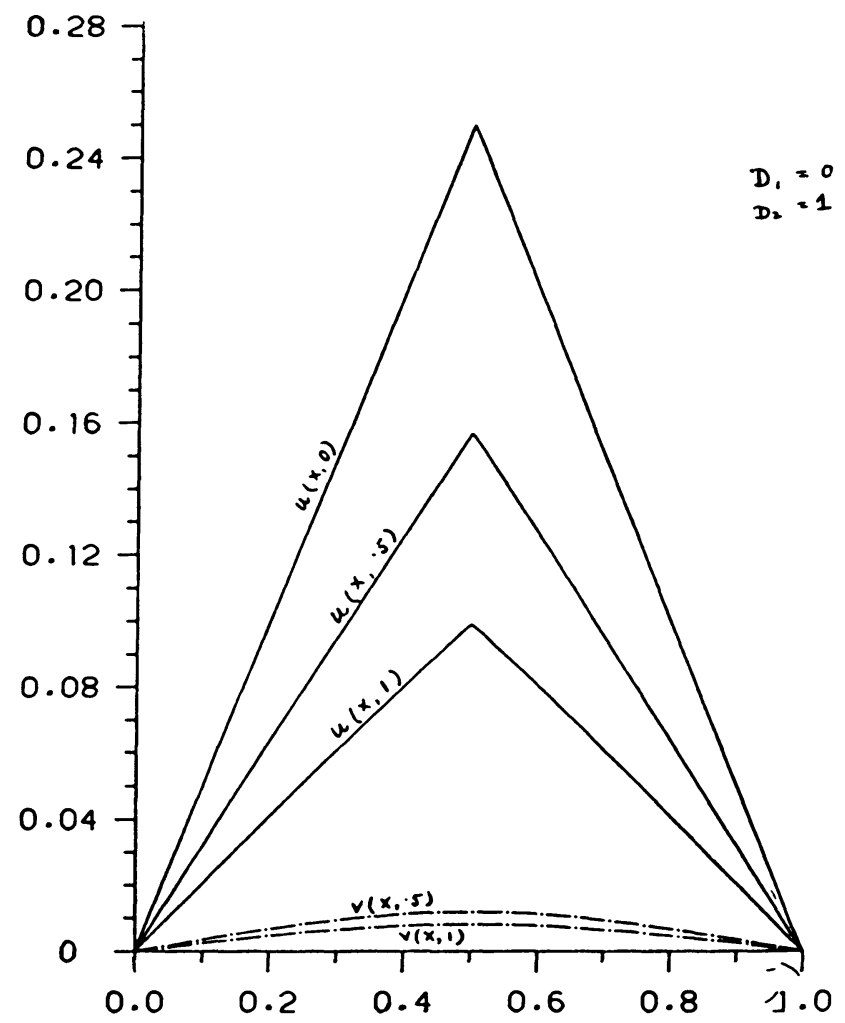

FIG. 1. DECAY OF DISCONTINUITY IN $\frac{\partial u}{\partial x}$ at $x=0.5$.

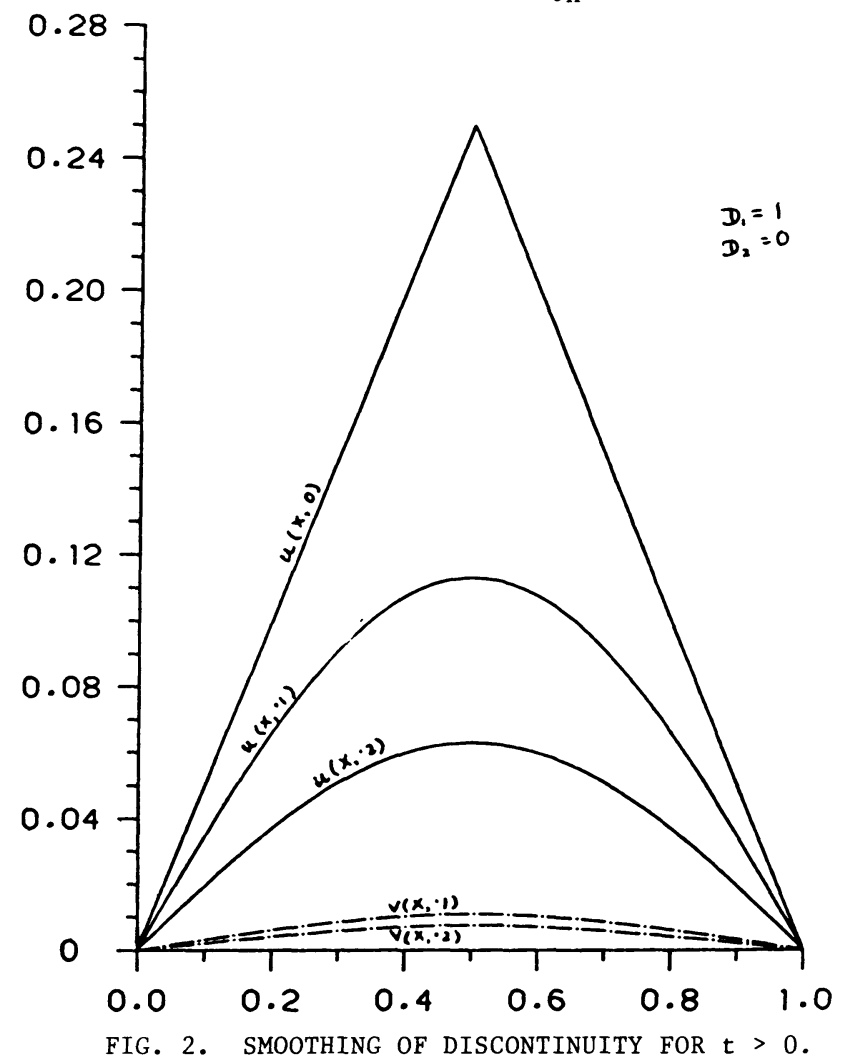

FIG. 2. SMOOTHING OF DISCONTINUITY FOR $\mathrm{t}>0$. 

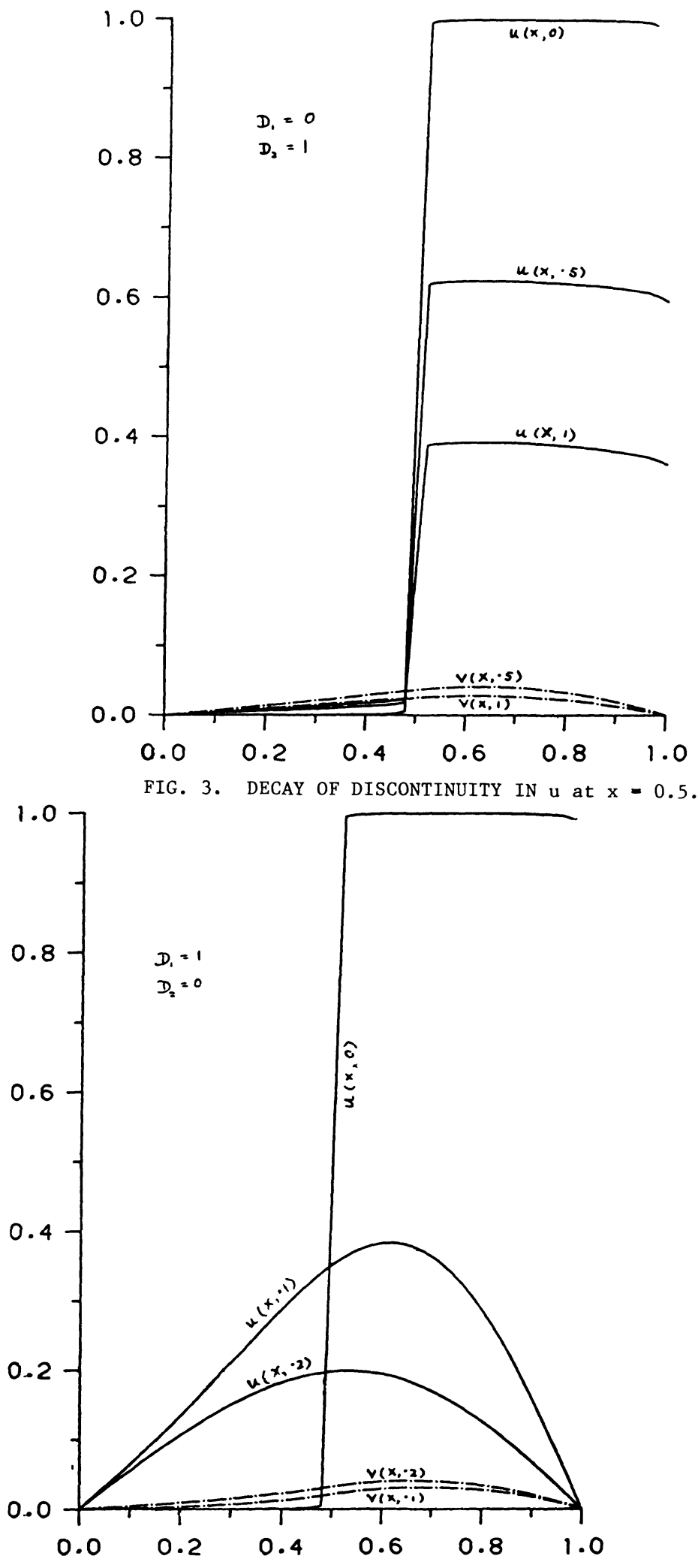

FIG. 4. DISCONTINUITIES ARE SMOOTHED OUT. 


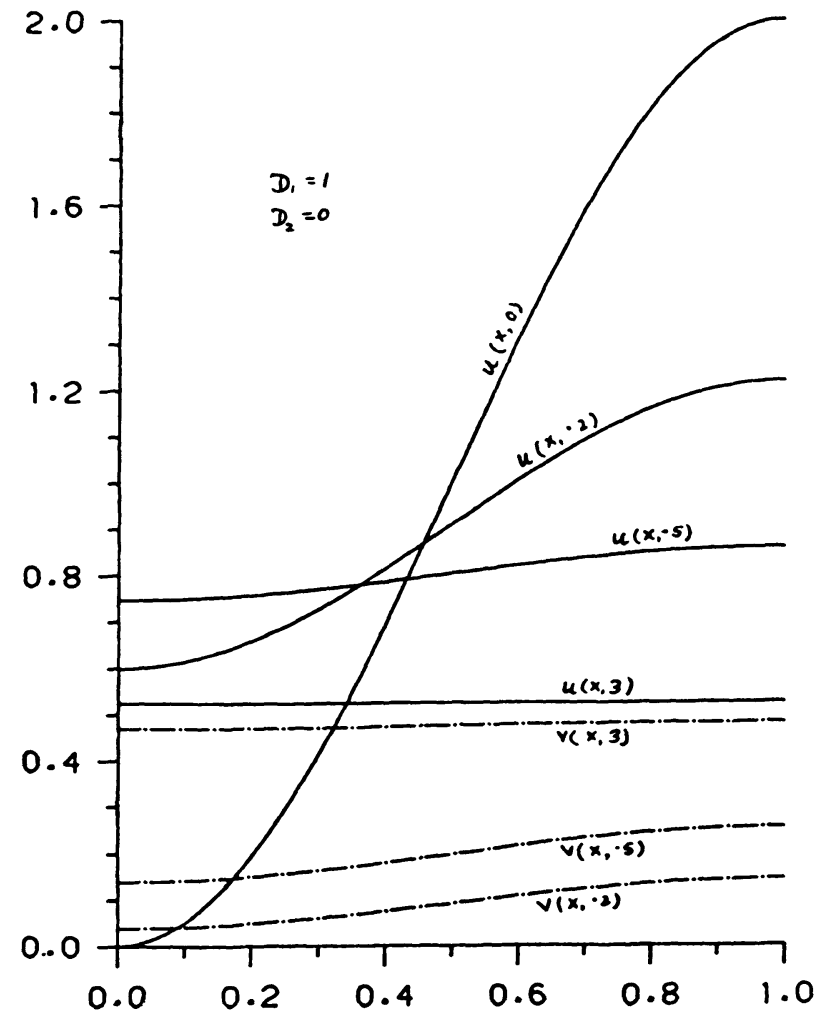

FIG. 5. $u \rightarrow \frac{1}{2}, v \rightarrow \frac{1}{2}$ AS $t \rightarrow \infty, D_{1}=0, D_{2}=1$.

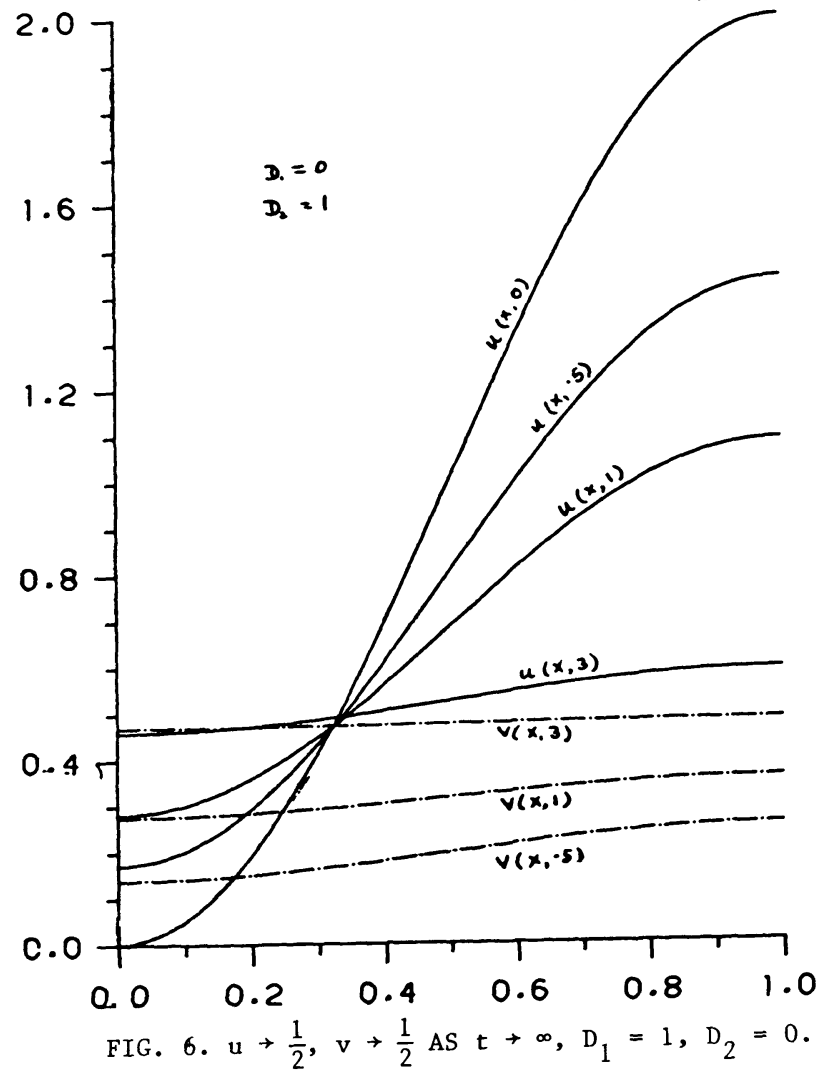




\section{REFERENCES}

[1] Barenblatt, G.I., Zhetlov, Iu. P. and Kochina, I.N., Basic concepts in the theory of seepage of homogeneous liquids in fissured rucis, Appl. Math. Mechs. 24, $1286-1303(1960)$.

[2] Chen, P.J. and Gurtin, M.E., On a theory of heat conduction involving two temperatures, ZAMP 19, 614-627 (1968).

[3] Hill, J.M., On the solution of Reaction-Diffusion equations, I.M.A. Journal of Appl. Math. 27, 177-194 (1981).

[4] Aifantis, E.C. and Hill, J.M., On the theory of Diffusion in media with double diffusivity I, Quar. Jour. Mech. \& Appl. Math. 23, 1-21 (1980).

[5] Hill, J.M. and Aifantis, E.C., On the theory of Diffusion in media with double diffusivity II, Quar. Jour. Mech. \& Appl. Math. 23, 23-41 (1980).

[6] Lee, A.I. and Hill, J.M., On the solution of Boundary Value Problems for Fourth Order Diffusion, Acta Mechanica 46, 23-35 (1983).

[7] Gopalsamy, K. and Aggarwala, B.D., On the non-existence of periodic solutions of the Reactive-Diffusive Volterra system of equations, Jour. Theor. Biol. $\underline{82}$, 537-540 (1980).

[8] Gradshteyn, I.S. and Ryzhik, I.M., Table of Integrals, Series and Products, Academic Press (1965). 


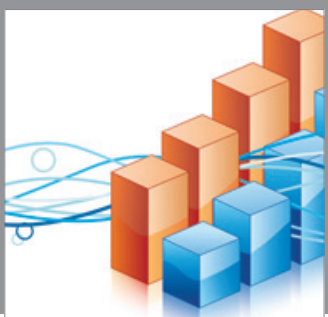

Advances in

Operations Research

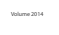

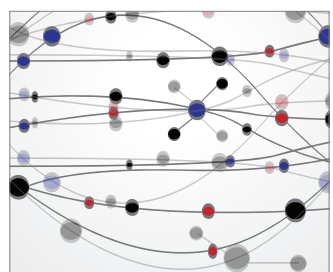

\section{The Scientific} World Journal
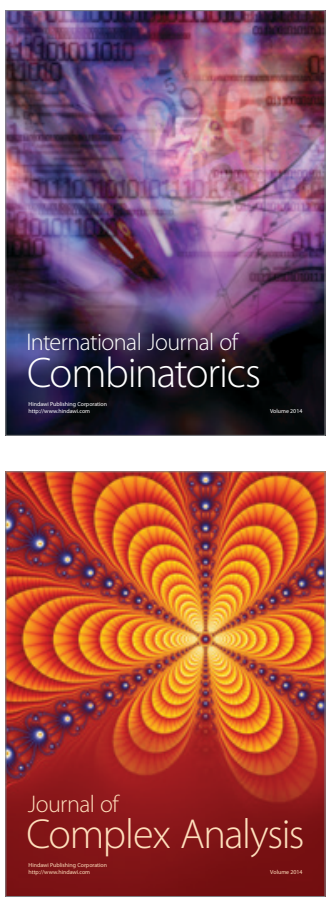

International Journal of

Mathematics and

Mathematical

Sciences
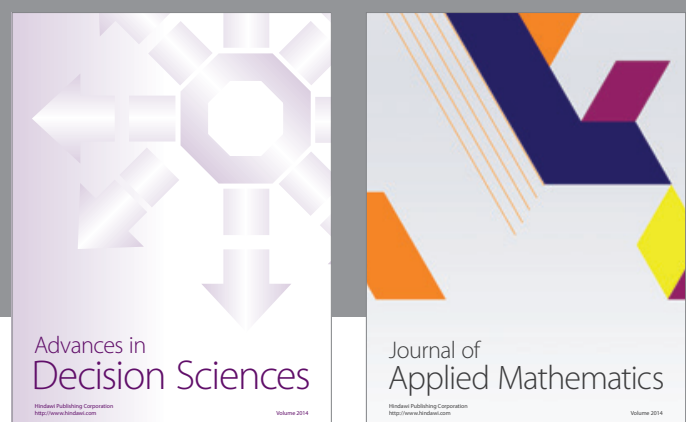

Journal of

Applied Mathematics
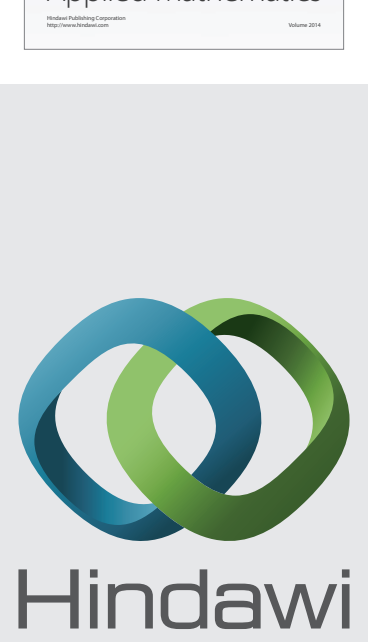

Submit your manuscripts at http://www.hindawi.com
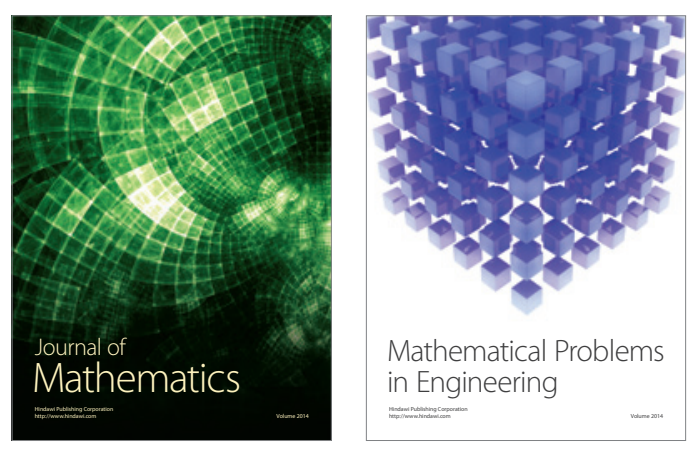

Mathematical Problems in Engineering
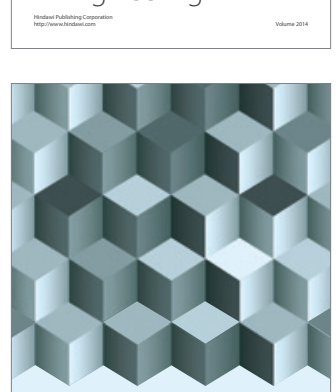

Journal of

Function Spaces
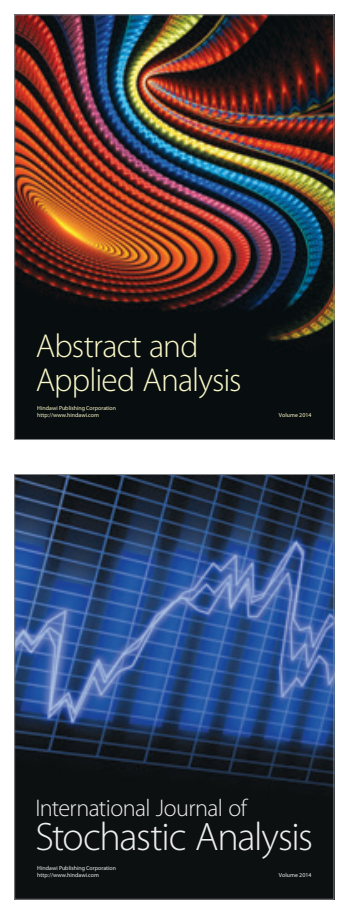

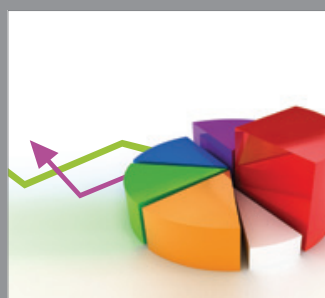

ournal of

Probability and Statistics

Promensencen
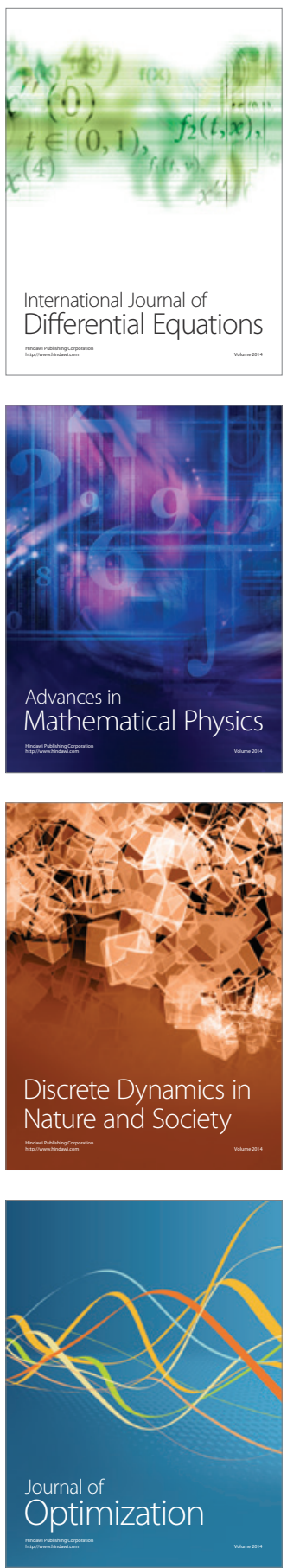